\title{
Trigeminal Neuralgia in Aqueduct Stenosis
}

\author{
WILLIAM S. TUCKER, ROSS FLEMING, FERELITH A. TAYLOR, and HART SCHUTZ
}

SUMMARY: Trigeminal neuralgia was the presenting symptom in two patients with aqueduct stenosis, hydrocephalus, and raised intracranial pressure. Treatment of the hydrocephalus resulted in the remission of pain in both patients.

RESUMÉ: Il est connu que dans certains cas les névralgies du trijumeau peuvent être symptomatiques d'une lésion démontrable, le plus souvent une masse de la fosse postérieure ou une anomalie vasculaire. Les cas présents réfërent à une névralgie du trijumeau clairement en rapport avec une pression intracrânienne augmentée, secondaire à une sténose de l'acqueduc. Nous n'avons pas retrouvé de cas semblable dans la littérature anglaise.

From the Divisions of Neurosurgery, Toronto Western and St. Michael's Hospitals, and the Department of Surgery, University of Toronto, Canada.

Reprint requests to: Dr. Hart Schutz, Toronto Western Medical Building, 25 Leonard Avenue, Toronto, Ontario, Canada M5T 2R2.
Some cases of trigeminal neuralgia are symptomatic of a demonstrable lesion, most commonly a posterior fossa mass or vascular abnormality (Seeger, 1963; White and Sweet, 1969). The present cases deal with trigeminal neuralgia related to chronic increased intracranial pressure (ICP), secondary to aqueduct stenosis. We have not found reports of this association in the English literature.

\section{CASE REPORTS}

Case 1

A 29-year-old woman presented with third division trigeminal neuralgia. Sharp jabbing pain in the jaw had occurred with increasing frequency over the previous three years. The pain was triggered by washing, chewing, or light contact with the lower lip. Remissions became shorter, and symptomatic periods were more severe and prolonged. Carbamazepine provided relief of symptoms. She denied headache, and was actively employed as an assembly worker.

Examination: The patient was obese, weighed $109 \mathrm{Kg}$., and was $170.5 \mathrm{~cm}$. tall. There was no deficit of trigeminal nerve function and the remainder of the neurological examination was normal. Psychometric testing suggested a 'brightnormal' intellectual capacity, with some superimposed intellectual impairment due to slowness of thought and psychomotor processes.

Investigations: Skull $\mathrm{x}$-rays showed evidence of chronic raised intracranial pressure, with erosion of the posterior clinoid processes and the dorsum sellae, and a 'copper-beaten' appearance to the entire cranial vault (Fig. 1). The EEG and radionuclide brain scan were normal. A right carotid arteriogram showed symmetrical ventricular enlargement. The right lateral ventricle was cannulated and continuous monitoring of the ICP was carried out for three days according to the technique described by Lundberg (1960). The base line ICP was moderately elevated at
$15 \mathrm{~mm} . \mathrm{Hg}$ (upper limit of normal $=10$ $\mathrm{mm} . \mathrm{Hg}$ ). There were numerous pressure waves recorded with a dominating frequency of 1 per minute and an amplitude of $18-40 \mathrm{~mm}$. $\mathrm{Hg}$. The recording appeared to be more active during sleep (Fig. 2). Following this, a positive contrast ventriculogram was performed, which showed gross enlargement of the lateral and third ventricles, with complete obstruction to the contrast medium in the aqueduct (Fig. 3). A subsequent pneumoencephalogram showed the fourth ventricle to be normal in size and position, but air did not rise beyond the mid portion of the aqueduct. Superimposition of the ventriculogram and pneumoencephalogram suggested a four $\mathrm{mm}$. length of stenosis of the aqueduct. The pneumoencephalogram also demonstrated some filling of the cerebral sulci. Cisternography with radio-iodinated serum albumin showed some circulation of isotope over the cerebral convexities but no ventricular reflux. During these latter investigations, the patient began to experience nocturnal incontinence, but on carbamazepine was free of tic pain.

Treatment: In September, 1973, a right ventriculo-cisternal shunt was performed. Carbamazepine was not re-ordered postoperatively and there was no recurrence of pain for one week. Then the patient began to experience the return of tic pain and nocturnal urinary incontinence. The tic pain responded to carbamazepine as before but the patient developed headache and repeated syncopal episodes. The suboccipital wound began to bulge and a repeat CSF scan was performed, injecting the isotope into the flushing device of the shunt. The tracer reached the cisterna magna, and refluxed into the fourth ventricle, but there was no circulation over the cerebral hemispheres. It was thought that the ventriculo-cisternal shunt was functioning, but that the absorptive capacity of the subarachnoid pathways was inadequate. In October, 1973, the shunt was revised to a ventriculoperitoneal shunt. Carbamazepine was stopped and at the latest follow-up $\left(4 \frac{1}{2}\right.$ years later) there has been no recurrence of pain. 


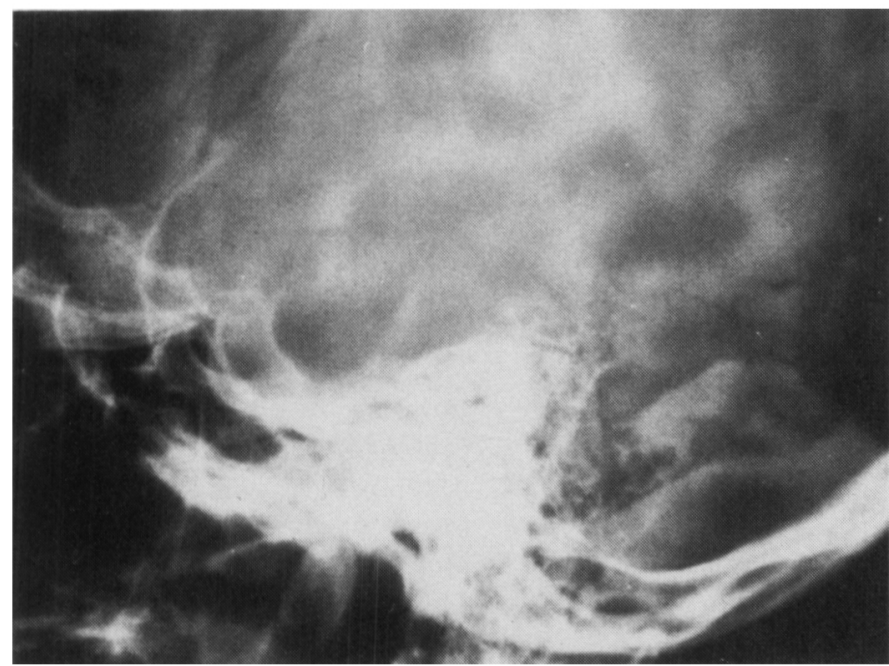

Figure 1-Plain skull x-ray of Case 1, showing changes of longstanding raised intracranial pressure.

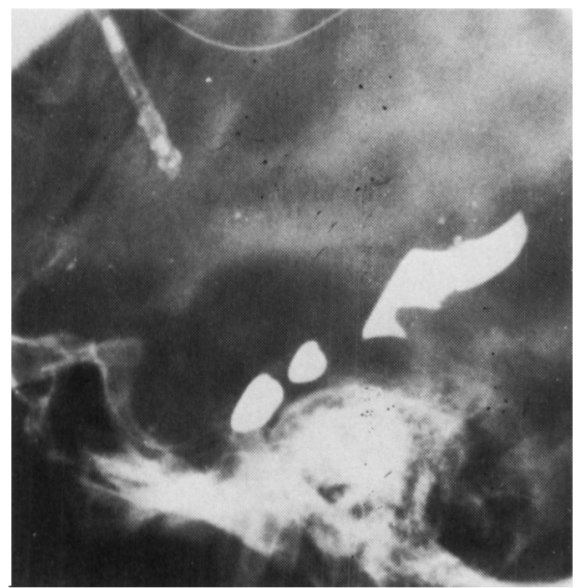

Figure 3-Positive contrast ventriculogram for Case 1, showing aqueduct stenosis.

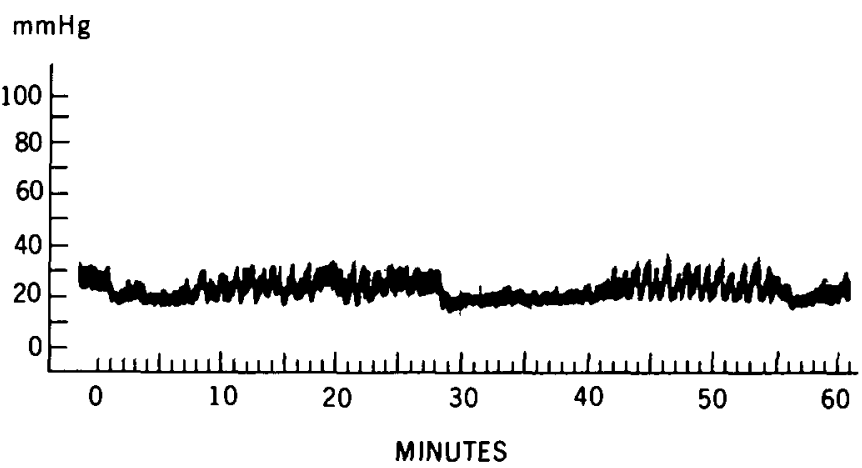

Figure 2-Tracing of ICP monitoring for Case 1, showing elevated base line pressure and intermittent pressure waves occurring during sleep.

\section{Case 2}

A 24-year-old man presented in 1958 with left sided trigeminal neuralgia. Over the next ten years he suffered intermittently from attacks of severe tic pain, with some spontaneous remissions. In 1962, he was treated with dilantin, which gave relief for several years. In 1969, during a bout of severe pain, carbamazepine was prescribed and this gave gratifying but incomplete relief of pain. The pain was of typical lancinating quality and involved all three trigeminal divisions on the left, but particularly the second division. Paroxysms were precipitated by talking, chewing, brushing the teeth, or touching the face. The patient had not suffered from headaches.
Examination: The patient's head appeared slightly larger than normal but there were no neurological abnormalities.

Investigations: Skull $\mathrm{x}$-rays showed sellar enlargement and thickening of the cranial vault with a 'copper-beaten' appearance (Fig. 4). A positive contrast ventriculogram showed aqueduct stenosis (Fig. 5). Unfortunately ICP monitoring was not carried out in this patient.

Treatment: A ventriculoperitoneal shunt was inserted in September, 1976. Following this, the trigeminal neuralgia was very much improved, though not completely relieved. Pain recurred at the time of subsequent malfunction of the shunt, which was revised in March, 1977, and the tic was again improved, and then cleared entirely.

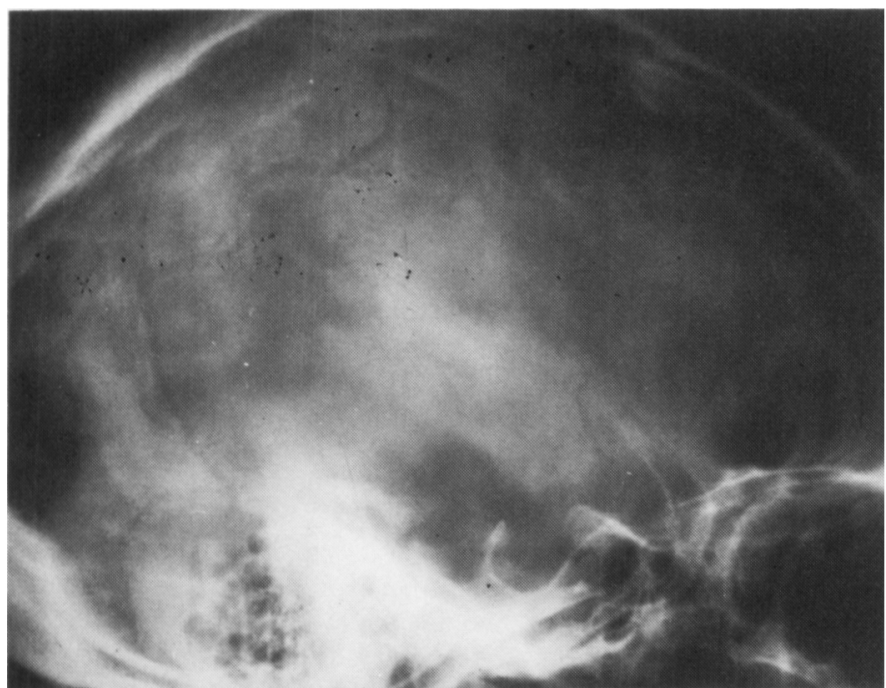

Figure 4-Plain skull $x$-ray of Case 2, showing the changes of partially arrested hydrocephalus and long-standing raised intracranial pressure.

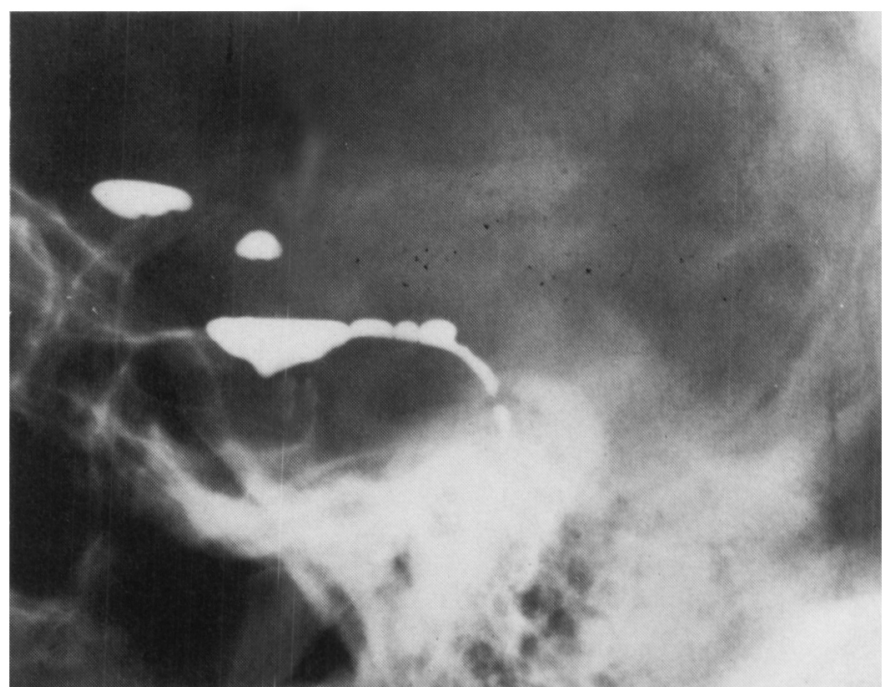

Figure 5-Positive contrast ventriculogram for Case 2, showing aqueduct stenosis. 
The patient has had no further tic pain since last seen in November 1977 (8 months) and immediately prior to the insertion of the shunt, this man was suffering from severe tic pain. He experienced a dramatic improvement in his symptoms at the time of shunting, and except for a temporary relapse associated with shunt dysfunction, the improvement has continued over the subsequent $1 \frac{1 / 2}{2}$ years.

\section{DISCUSSION}

There seemed to be a definite relationship between the first patient's tic pain and the level of the ICP. The ICP was elevated prior to the operation and during the interval between the first and second operations. At these times, the pain could only be managed with carbamazepine. Since the relief of the intracranial hypertension by shunting, the patient has had no further symptoms. The response to shunting in the second patient was equally dramatic, although the very long history of tic pain has rendered the patient somewhat cautious and reserved in his enthusiasm.

Numerous causes of symptomatic trigeminal neuralgia have been reported (Seegar, 1963; White and Sweet, 1969; Jannetta, 1974), and in most cases there has been distortion of a part of the trigeminal root by a lesion. Dandy (1934), in his large series of posterior fossa root sections for trigeminal neuralgia, found structural lesions related to the trigeminal root in $10.7 \%$ of cases. He encountered acoustic neuromas, pearly tumors, osteomas of the base of the skull, basilar artery aneurysms, and angiomas. Dandy also thought that impingement on the trigeminal root by arteries and veins was of etiologic significance in tic douloureaux, a concept recently stressed by Jannetta (1974). Hamby (1947) pointed out that posterior fossa tumors causing tic could be contralateral to the pain and thus the association need not depend on direct contact of the lesion with the involved trigeminal pathway, but may be based on distortion of the trigeminal system by more remote lesions. Gardner et al. (1956) described asymmetry of the petrous ridges in a number of patients suffering from trigeminal neuralgia and found that trigeminal neuralgia was commoner on the side of the higher petrous ridge, as determined radiographically. However, radiographs revealed symmetric petrous ridges in our patients, so this factor did not predispose the patients to tic. Seeger (1963) documented three cases in which tic pain was related to obstructive hydrocephalus on the basis of aqueduct stenosis, colloid cyst, and a suprasellar craniopharyngioma, respectively. The cause of tic pain associated with lesions remote from the trigeminal root, or raised ICP, is not clear. There may be a shift of the brainstem due to pressure gradients between supra and infra tentorial compartments, resulting in compression of the trigeminal nerve root.
In our cases there was no local structural lesion distorting the trigeminal root and the pain appeared to be directly related to raised ICP. It is interesting to speculate that the onset and exacerbations of tic in these patients might have been precipitated by a degree of decompensation in the long-standing hydrocephalus. Control of the ICP with adequate shunting has resulted in marked improvement in the symptoms of trigeminal neuralgia in both patients.

\section{REFERENCES}

DANDY, W. E. (1934). Concerning the cause of trigeminal neuralgia. American Journal of Surgery, 24, 447-455.

GARDNER, W. J., TODD, E. M. and PINTO, J. P. (1956). Roentgenographic findings in trigeminal neuralgia. American Journal of Roentgenology, 76, 346-350.

HAMBY, W. B. (1947). Trigeminal neuralgia due to contralateral tumors of the posterior fossa. Journal of Neurosurgery, 4, 179-182.

JANNETTA, P. J. (1974). Tic douloureaux and facial spasm. Letter to the Editor. Journal of the American Medical Association, 228, 1637-8.

LUNDBERG, N. (1960). Continuous recording and control of ventricular fluid pressure in neurosurgical practice. Acta Psychiatrica Scandinavia, 36, Supplement 149, 1-193.

SEEGER, W. (1963). Trigeminusneuralgie bei raumfordernden intrakraniellen Prozessen. Zbl. Neurochirurgie, 23, 152-165.

WHITE, J. C. and SWEET, W. H. (1969). Pain and the Neurosurgeon: A Forty Year Experience. 129-147. Charles C. Thomas, Springfield, Illinois. 